\title{
Past and present in neonatal exchange transfusion
}

The exchange transfusion (ET) contributed to reduce infant mortality and prevent kernicterus in severe neonatal hyperbilirubinemia. As we reach the 70 years anniversary with the use of ET and considering how infrequent is today this procedure in our nurseries. I consider a good opportunity to remember our experience with the geniality of this treatment. Consequently, the history of the ET through the umbilical vein ${ }^{1}$ has a strong connection with modern Neonatology. The use of the umbilical vein was an extraordinary milestone in the care of the newborn and became essential in cardiopulmonary resuscitation, respiratory distress syndrome, sepsis and many other neonatal diseases. Likewise its use allowed the administration of physiologic solution, blood, drugs, central venous pressure measurement and cardiac catheterization.

Dr. Louis Diamond was the first physician in utilizing the umbilical vein in the ET technique, ${ }^{2}$ in October 1946 at the Boston Lying-in Hospital, a procedure with enormous historical influence in Neonatology. The purpose of the ET is to reduce the levels of serum unconjugated bilirubin, potentially toxic to the brain, correct the anemia, replacing "sensitized" RBC by healthy ones, remove anti -Rh IGG antibodies and also maternal anti A and anti B from newborn blood, to reduce the product of hemolysis in erythroblastosis fetalis (EF), ABO incompatibility and GP6D deficiency. The removal of products of hemolysis during the disease process ameliorate the decrease in albumin binding capacity and reduces the levels of "free" bilirubin toxic to the brain. I remember that as a pediatric resident during my rotations through Maternidad Sarda Hospital, in Buenos Aires, we followed the climbing of serum bilirubin in $\mathrm{mg}$ /hour and we made the correlations with the Diamond's curve of bilirubin. ${ }^{2}$ During the 1970 decade, using this curve we were able to prevent kernicterus, success also linked to the measurement of high maternal anti-Rh titer, history of severe hemolytic disease and other risk factors; and also levels of hemoglobin, bilirubin and reticulocytes in umbilical cord blood.

Diamond observed that $80-90 \%$ of the newborns with EF and levels of bilirubin $\geq 40$ $\mathrm{mg} / \mathrm{dl}$ developed kernicterus, $50 \%$ when they had levels $\geq 30 \mathrm{mg} / \mathrm{dl}$ and there were no cases of kernicterus among 200 studied newborns whose serum bilirubin were $<20 \mathrm{mg} / \mathrm{dl} .{ }^{2}$ With the ET and the use of the Diamond's curve, pediatricians and neonatologists in the whole world, observed the disappearance of kernicterus for over 20 years. Without doubt that phototherapy and the anti-D gammaglobulins to $\mathrm{Rh}$ negative mothers were also contributor factors.

Unfortunately, several countries begun to have new cases in 1991. In USA there are several factors incriminated for this "backward step" and the resurgence of kernicterus. In my judgement there were many errors such as the critic of the "vigintophobia", or phobia of the $20 \mathrm{mg} / \mathrm{dl}$, the ignorance of all the research and experience of Dr. Diamond, the ignorance of all research published over the toxicity of bilirubin, the failure to recognize the risk factors, the "shorter" hospital stay after birth, health plans and insurance companies that did not cover the cost of hospitalization and ignorance of correct breast feeding practices. In addition we had a new generation of medical residents and pediatricians not familiarized with erythroblastosis and much less in recognizing the "dangers "of severe hyperbilirubinemia. If all of these were not enough, the Guidelines of the American Academy of Pediatrics (AAP) in 1994 recommended the ET in low risk newborns with serum bilirubin levels $\geq 30 \mathrm{mg} / \mathrm{dl}$.

In a recent revision of the technique, equipment and precautions of the $\mathrm{ET}^{3}$ we did not observed a substantial difference compared with the one described by Diamond in $1950 .^{2}$ Today we use a disposable ET tray that practically contains all the needed instruments, catheters, Luer-Lok syringes,"4-way" stopcocks key, extension tubing sets and a waste blood bag (almost identical to the one described by Diamond in the original technique). One difference is the Blood Warmer for donor blood we use in our hospital; in many hospitals the Blood Bank provides the donor blood already warmed.

In the 60's and 70's decades, the ET was a very common treatment in our maternities and NICU's, two or three ET's per night was the usual practice. Then with the discovery of the anti-D gammaglobulins, the use of phototherapy and a better prenatal care, we observed the progressive disappearance of the ET from our nurseries. The statistics of the Good Samaritan NICU Hospital in Cincinnati, Ohio, has shown 32 ET per week in the 1960's, an average of 200 per year in the 1970's and at present we do 1 or 2 per year. As a result very few pediatric residents and fellows 
in Neonatology can acquire enough experience to perform an ET.

Another factor which contributed to the decrease in the number of ET has been the administration of intravenous immuneglobulins (IVIG).The AAP Guidelines on Hyperbilirubinemia in $2004^{4}$ recommended the administration of IVIG in erythroblastosis (selective cases) if the total serum bilirubin (TSB) is rising despite intensive phototherapy or the TSB level is within 2-3 $\mathrm{mg} / \mathrm{dl}$ of the exchange level. Even though the treatment could be effective by blocking antibodies receptors in RBC's, ameliorating hemolysis and bilirubin production, the results have not been universally positive. Two recent publications, in Ankara, Turkey in $2011^{5}$ and Rio de Janeiro, Brasil in 2013, ${ }^{6}$ have shown that the administration of IVIG was not effective in reducing the need for ET in EF and $\mathrm{ABO}$ incompatibility. In my personal experience with ET I remember that in many opportunities like in newborns with cephalohematoma or G6PD deficiency, the IVIG was not effective in avoiding an ET. There are not randomized controlled trials where the randomization method is clear, comparing the efficacy of ET versus IVIG. The initial enthusiasm for IVIG could be due to its simplicity of ordering and administering it, compared with the relative complexity of the ET and specially due to the obvious lack of experience by our current neonatologists.

Therefore, I don't believe, the ET as a treatment will disappear from our services and becomes obsolete.

The tradition of "see one ET, do one, and teach

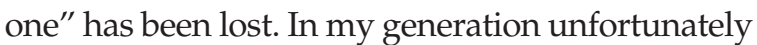
there were very few randomized control trials in Neonatology and Evidence Based Medicine did not exist; yet generations of residents, physicians and nurses were able to share the "pearls and pitfalls" of the ET that in our country saved the lives of thousands of newborns. Many of these pearls and pitfalls were never published and they only remain in our memory. Any pediatric resident who was lucky to work or rotate through Maternidad Sarda or Hospital de Niños Ricardo Gutierrez will be able to recognize easily these facts.

Today, even though the technique of the ET is easy to teach, the details of the procedure are many ${ }^{3}$ and the lack of skills by the Neonatology team can lead to serious complications for the newborn. This reality is extended to all hospital levels including the departments of Nursing, Obstetrics, Radiology, Laboratory, Hematology, Blood Bank and others. The need for an ET today, produces a "total chaos" in our NICU's. This chaotic situation results from our ignorance of not knowing, for example, how to initiate an ET, where is the equipment that we will need and how are the connections between the keys, the waste blood bag, the donor blood and the infant. Likewise, from the "stress" we have knowing the nurse who is going to help us has never seen an ET. This will increase the risk of complications associated to the procedure.

Fortunately the etiology of severe hyperbilirubinemia in 2015 is very much different than in 1968 when severe Rh issoimmunization was predominant. Thus, mortality and morbidity from ET prior to 1990 was much higher and after 1990 the most common complications were: thrombocytopenia in $44 \%$,hypocalcemia in $29 \%$ and metabolic acidosis in $24 \%,{ }^{3}$ easily corrected with effective treatments. Mortality from ET today is $1 \%{ }^{7}$ We recommend the use of a simulation lab with mock trials similar to the ones we perform in Neonatal Resuscitation Program, to know and practice the connections between the key, blood donor and patient. A poster with photographs of the keys and connections can also facilitate the learning of the technique. These aspects were described in detail in Neo Reviews and also in Proneo, Update Program in Neonatology ${ }^{3,7}$ which are very useful for the on call pediatric resident and neonatologist in preparing for an ET. Recently in 2013,the St Louis Children's Hospital in Missouri, published two instructional videos: Catheterization of the umbilical vein ${ }^{8}$ and Technique of the ET. ${ }^{9}$

Finally, I insist that is indefectible the ET be performed by a neonatologist with enough experience in the technique and possible complications. Only then, we will be able to achieve a safe treatment.

Acknowledgement: The author thank Dr. José M. Ceriani Cernadas, Editor of "Archivos Argentinos de Pediatría", for his selfless help and his thoughtful review of this manuscript.

Horacio S. Falciglia, M.D.

Neonatologist, Children's Hospital of Cincinnati, Cincinnati, Ohio. USA.

Profesor Emeritus of Pediatrics, School of Medicine, University of Cincinnati, Cincinnati, Ohio. National Honorary Member, Argentine Pediatric Society International Editorial Committee Member, Archivos Argentinos de Pediatria

http:/ /dx.doi.org/10.5546/aap.2016.eng.191 


\section{REFERENCES}

1. Philip AG, Diamond LK, Pearson HA. Historical perspectives: The rise and fall of exchange transfusion. Neoreviews 2003;4(7):e169-74.

2. Allen FH Jr, Diamond LK. Erythroblastosis fetalis including exchange transfusion technique. Boston: Little, Brown and Company; 1957.Pág.57.

3. Falciglia HS, Greenwood CS. Double volume exchange transfusion: a review of the "Ins and Outs". Neoreviews 2013;14(10):e513-20.

4. American Academy of Pediatrics Subcommittee on Hyperbilirubinemia. Management of hyperbilirubinemia in the newborn infant 35 or more weeks of gestation. Pediatrics 2004;114(1):297-316.

5. Demirel G, Akar M, Celik IH, Erdeve O, et al. Single versus multiple dose intravenous immunoglobulin in combination with LED phototherapy in the treatment of $\mathrm{ABO}$ hemolytic disease in neonates. Int J Hematol 2011;93(6):700-3.

6. Santos MC, Sa C, Gomes SC Jr, Camacho LA, et al. The efficacy of the use of intravenous human immunoglobulin in Brazilian newborns with rhesus hemolytic disease: a randomized double-blind trial. Transfusion 2013;53(4): 777-82.

7. Falciglia HS. Exanguinotransfusión en el recién nacido. [Curso virtual]. Programa de Actualización en Neonatología PRONEO.Séptimo Ciclo, Modulo3. Editorial Panamericana; 2007.Págs.113-40.

8. Moeckel D, Cresalia N, Vachharajani A. Umbilical vein catheterization. Neoreviews 2013;14(8):e416-8.

9. Moeckel D, Julian S, Vachharajani AJ. Double volume exchange transfusion. Neoreviews 2013;14(10):e536-8. 